\title{
Revisiting the optimal detection of quantum information
}

\author{
Eric Chitambar ${ }^{1, *}$ and Min-Hsiu Hsieh ${ }^{2, \dagger}$ \\ ${ }^{1}$ Department of Physics and Astronomy, Southern Illinois University, Carbondale, Illinois 62901, USA \\ ${ }^{2}$ Centre for Quantum Computation \& Intelligent Systems (QCIS), Faculty of Engineering and Information Technology (FEIT), \\ University of Technology, Sydney (UTS), NSW 2007, Australia
}

(Received 1 May 2013; published 27 August 2013)

\begin{abstract}
In 1991, Peres and Wootters wrote a seminal paper on the nonlocal processing of quantum information [Phys. Rev. Lett. 66, 1119 (1991)]. We return to their classic problem and solve it in various contexts. Specifically, for discriminating the "double trine" ensemble with minimum error, we prove that global operations are more powerful than local operations with classical communication (LOCC). Even stronger, there exists a finite gap between the optimal LOCC probability and that obtainable by separable operations (SEP). Additionally we prove that a two-way, adaptive LOCC strategy can always beat a one-way protocol. Our results demonstrate "nonlocality without entanglement" in two-qubit pure states.
\end{abstract}

DOI: 10.1103/PhysRevA.88.020302

PACS number(s): 03.67.Mn, 03.65.Ud

One physical restriction that naturally emerges in quantum communication scenarios is nonlocality. Here, two or more parties share some multipart quantum system, but their subsystems remain localized with no "global" quantum interactions occurring between them. Instead, the system is manipulated through local quantum operations and classical communication (LOCC) performed by the parties.

Peres and Wootters were the first to introduce the LOCC paradigm and study it as a restricted class of operations in their seminal work [1]. To gain insight into how the LOCC restriction affects information processing, they considered a seemingly simple problem. Suppose that Alice and Bob each possess a qubit, and with equal probability, their joint system is prepared in one of the states belonging to the set $\left\{\left|D_{i}\right\rangle=\right.$ $\left.\left|s_{i}\right\rangle \otimes\left|s_{i}\right\rangle\right\}_{i=0}^{2}$, where $\left|s_{i}\right\rangle=U^{i}|0\rangle$ and $U=\exp \left(-\frac{i \pi}{3} \sigma_{y}\right)$. This highly symmetric ensemble is known as the "double trine," and we note that lying orthogonal to all three states is the singlet $\left|\Psi^{-}\right\rangle=\sqrt{1 / 2}(|01\rangle-|10\rangle)$.

Alice and Bob's goal is to identify which double trine element was prepared only by performing LOCC. Like any quantum operation used for state identification, Alice and Bob's collective action can be described by some positiveoperator valued measure (POVM). While the nonorthogonality of the states prohibits the duo from perfectly identifying their state, there are various ways to measure how well they can do. Peres and Wootters chose the notoriously difficult measure of accessible information [2], but their paper raises the following two general conjectures concerning the double trine ensemble, which can apply to any measure of distinguishability: (C1) LOCC is strictly suboptimal compared to global operations. (C2) The optimal LOCC protocol involves two-way communication and adaptive measurements.

The set of global POVMs will be denoted by GLOBAL, and $\mathrm{C} 1$ can be symbolized by GLOBAL $>$ LOCC. A two-way LOCC protocol with adaptive measurement refers to at least three rounds of measurement, Alice $\rightarrow$ Bob $\rightarrow$ Alice, with the choice of measurement in each round depending on the

\footnotetext{
*echitamb@siu.edu

†Min-Hsiu.Hsieh@uts.edu.au
}

outcome of the other party's measurement in the previous round. We symbolize C2 as LOCC $>$ LOCC $_{\rightarrow}$. In Ref. [1] Peres and Wootters obtained numerical data to support both $\mathrm{C} 1$ and $\mathrm{C} 2$, but these conjectures have never been proven for the double trine.

Before we present our contribution to the problem, we would like to briefly highlight the legacy of the Peres-Wootters paper. Perhaps most notably is that it subsequently led to the discovery of quantum teleportation [3]. Other celebrated phenomena can also directly trace their roots to Ref. [1] such as so-called nonlocality without entanglement [4] and quantum data hiding [5]. More generally, Ref. [1] paved the way for future research into LOCC and its fundamental connection to quantum entanglement [6].

We finally note that in a return to Ref. [1] of his own, Wootters constructed a separable POVM that obtains the same information as the best-known global measurement [7]. A POVM $\left\{\Pi_{i}\right\}$ belongs to the class of separable operations (SEP) if each POVM element can be decomposed as a tensor product $\Pi_{i}=A_{i} \otimes B_{i}$ over the two systems. SEP is an important class of operations since every LOCC operation belongs to SEP [4].

In this Rapid Communication, we prove that conjectures $\mathrm{C} 1$ and $\mathrm{C} 2$ are indeed true when distinguishability success is measured by the minimum error probability, which is defined as follows. For an ensemble $\mathcal{E}=\left\{\left|\psi_{i}\right\rangle, p_{i}\right\}_{i=1}^{k}$, the error probability associated with some identification POVM $\left\{\Pi_{i}\right\}_{i=1}^{k}$ is given by $1-\sum_{i=1}^{k} p_{i}\left\langle\psi_{i}\left|\Pi_{i}\right| \psi_{i}\right\rangle$. Then the minimum error probability of distinguishing $\mathcal{E}$ with respect to a class of operations $\mathcal{S}$ (such as LOCC, SEP, GLOBAL, etc.) is given by the infimum of error probabilities taken over all POVMs that can be generated by $\mathcal{S}$. Note that we can replace "infimum" by "minimum" only if $\mathcal{S}$ is a compact set of operations. While GLOBAL, SEP, and $\mathrm{LOCC}_{\rightarrow}$ all have this property, LOCC does not $[8,9]$. Hence, to properly discuss the LOCC minimum error, we must consider the class of so-called asymptotic $L O C C$, which is LOCC plus all its limit operations [9]. We will prove $\mathrm{C} 1$ with respect to this more general class of operations.

Global and separable operations. The double trine ensemble has a group-covariant structure which greatly simplifies the analysis. In fact, Ban et al. have proven that the 
so-called "pretty good measurement" (PGM) [10] is indeed an optimal global POVM for discriminating ensembles with such symmetries [11]. For the double trine, the PGM consists of simply projecting onto the orthonormal basis $\left\{\left|\Psi^{-}\right\rangle,\left|F_{i}\right\rangle\right\}_{i=0}^{2}$, where

$$
\left|F_{i}\right\rangle \propto U^{i} \otimes U^{i}[(\sqrt{2}+1)|00\rangle-(\sqrt{2}-1)|11\rangle] .
$$

The corresponding error probability is

$$
1 / 2-\sqrt{2} / 3 \approx 2.86 \times 10^{-2} .
$$

To show that SEP can also obtain this probability, we explicitly construct a separable POVM. The idea is to mix a sufficient amount of the singlet state with each of the PGM POVM elements so as to obtain separability (a similar strategy was employed in Ref. [7]). The resulting POVM is $\left\{\left|\tilde{F}_{i}\right\rangle\left\langle\tilde{F}_{i}\right|\right\}_{i=0}^{2}$ with $\left|\tilde{F}_{i}\right\rangle\left\langle\tilde{F}_{i}|=| F_{i}\right\rangle\left\langle F_{i}|+1 / 3| \Psi^{-}\right\rangle\left\langle\Psi^{-}\right|$. It is fairly straightforward to compute that $\tilde{F}_{0}=1 / 2\left(\left|\varphi_{+}\right\rangle\left\langle\varphi_{+}\right|+\right.$ $\left.\left|\varphi_{-}\right\rangle\left\langle\varphi_{-}\right|\right)$, where $\left|\varphi_{ \pm}\right\rangle=\left|F_{0}\right\rangle \pm \sqrt{1 / 3}\left|\Psi^{-}\right\rangle$is a product state. This suffices to prove separability of the POVM.

LOCC and asymptotic LOCC. Let us begin with a clear description of asymptotic LOCC discrimination. In general, a sequence of POVMs $\mathcal{P}^{(n)}:=\left\{\Pi_{i}^{(n)}\right\}_{i=1}^{k}$ asymptotically attains an error probability $P$ on ensemble $\left\{\left|\psi_{i}\right\rangle, p_{i}\right\}_{i=1}^{k}$ if for every $\epsilon>0$ we have $P+\epsilon>1-\sum_{i=1}^{k} p_{i}\left\langle\psi_{i}\left|\Pi_{i}^{(n)}\right| \psi_{i}\right\rangle$ for sufficiently large $n$. If each POVM in the sequence $\mathcal{P}^{(n)}$ can be generated by LOCC, then $P$ is achievable by asymptotic LOCC.

It is known that for an ensemble of linearly independent pure states, the global POVM attaining minimum error consists of orthonormal, rank-one projectors [12] (see also [13]). We strengthen this result and extend it to the asymptotic setting.

Theorem 1. Let $\mathcal{E}=\left\{\left|\psi_{i}\right\rangle, p_{i}\right\}_{i=1}^{k}$ be an ensemble of linearly independent states spanning some space $S$. Suppose that $P_{\text {opt }}$ is the global minimum error probability of $\mathcal{E}$. Then there exists a unique orthonormal basis $\left\{\left|\phi_{i}\right\rangle\right\}_{i=1}^{k}$ of $S$ such that (a) a POVM attains an error probability $P_{\mathrm{opt}}$ on $\mathcal{E}$ if and only if it can also distinguish the $\left\{\left|\phi_{i}\right\rangle\right\}_{i=1}^{k}$ with no error, and (b) a sequence of POVMs asymptotically attains an error probability $P_{\text {opt }}$ on $\mathcal{E}$ if and only if it contains a subsequence that can asymptotically distinguish the $\left\{\left|\phi_{i}\right\rangle\right\}_{i=1}^{k}$ with no error.

The proof is given in the Supplemental Material [14]. Theorem 1 essentially reduces optimal distinguishability of nonorthogonal linearly independent ensembles to perfect discrimination of orthogonal ensembles. Applying part (a) to the double trine ensemble, if an LOCC POVM could attain the error probability of Eq. (2), then it can also perfectly distinguish the states $\left|F_{i}\right\rangle$ given by (1). In other words, the $\left|F_{i}\right\rangle$ are the $\left|\phi_{i}\right\rangle$ of Theorem 1. However, these are three entangled states which, by a result of Walgate and Hardy, means they cannot be distinguished perfectly by LOCC [15]. Therefore, the global minimum error probability is unattainable by LOCC.

But is the probability attainable by asymptotic LOCC? If it is, then part (b) of Theorem 1 likewise implies that the $\left|F_{i}\right\rangle$ must be perfectly distinguishable by asymptotic LOCC. While Ref. [15] provides simple criteria for deciding perfect LOCC distinguishability of two-qubit ensembles, no analogous criteria exists for asymptotic LOCC. The only general result for asymptotic discrimination has been recently obtained by Kleinmann et al. [16]. Here we cite their result in its strongest form, adapted specifically for the problem at hand.

Proposition 1. [16] If the states $\left\{\left|F_{i}\right\rangle\right\}_{i=0}^{2}$ can be perfectly distinguished by asymptotic LOCC, then for all $\chi \in$ $[1 / 3,1]$ there is a product operator $E \geqslant 0$ such that (i) $\sum_{i=0}^{2}\left\langle F_{i}|E| F_{i}\right\rangle=1$, (ii) $\left\langle F_{0}|E| F_{0}\right\rangle=\chi \geqslant\left\langle F_{i}|E| F_{i}\right\rangle$ for $i=$ 1,2 , and (iii) the normalized states $\left|F_{i}^{\prime}\right\rangle:=\frac{1}{\sqrt{\left\langle F_{i}|E| F_{i}\right\rangle}} E^{1 / 2}\left|F_{i}\right\rangle$ are perfectly distinguishable by separable operations.

In the Supplemental Material [14] we prove that these three conditions cannot be simultaneously satisfied; therefore, GLOBAL > LOCC for minimum error discrimination. Here, we provide a little intuition into why Proposition 1 must be true. For every LOCC protocol that correctly identifies the given state with probability $1-\epsilon$, we can think of the success probability as smoothly evolving from complete randomness $(\chi=1 / 3)$ to its final average value $(\chi=1-\epsilon)$. Then for each $\chi \in(1 / 3,1-\epsilon)$, the protocol can be halted after some sequence of measurement outcomes (collectively represented by the product operator $E$ ) such that given these outcomes: (1) there is one state that can be identified with probability $\chi$ (which by symmetry we can assume is $\left|F_{0}\right\rangle$ ), and (2) the transformed ensemble can be discriminated by a separable POVM with success probability no less than $1-\epsilon$. By compactness of SEP, we let $\epsilon \rightarrow 0$ and replace (2) by the condition that a separable POVM perfectly distinguishes the post-halted ensemble.

$L O C C>L O C C_{\rightarrow}$. We will now compute the minimum one-way error probability for the double trine, and then describe an explicit two-way protocol with a smaller error probability. In the one-way task, Alice makes a measurement and communicates her result to Bob. Without loss of generality, we fine-grain Alice's measurement so that each POVM element is rank one $|\eta\rangle\langle\eta|$, with $|\eta\rangle=r \cos \theta|0\rangle+r e^{i \phi} \sin \theta|1\rangle$. Given outcome $\eta$, Bob's task is to optimally discriminate the ensemble $\left\{\left|s_{i}\right\rangle\right\}_{i=0}^{2}$, but now with an updated distribution $\left\{p_{i}\right\}_{i=0}^{2}$ given by

$$
p_{k}=\frac{\left|\left\langle\eta \mid s_{k}\right\rangle\right|^{2}}{3 P(\eta)}=\frac{2}{3}\left|\cos \frac{2 \pi k}{3} \cos \theta+e^{i \phi} \sin \frac{2 \pi k}{3} \sin \theta\right|^{2} .
$$

Here, $P(\eta)=\frac{1}{3} \sum_{i=0}^{2}\left|\left\langle\eta \mid s_{i}\right\rangle\right|^{2}$, and we have used the covariance $\frac{1}{3} \sum_{i=0}^{2}\left|s_{i}\right\rangle\left\langle s_{i}\right|=\mathbb{I} / 2$. Additionally, we can assume that $p_{0} \geqslant p_{1}, p_{2}$, since if $|\eta\rangle$ fails to generate a distribution with this property, by the symmetry we can always rotate $|\eta\rangle$ such that $p_{0}$ is indeed the maximum post-measurement probability. This means we can only restrict attention to $-\pi / 6 \leqslant \theta \leqslant \pi / 6$.

Next, we observe that Bob's task of distinguishing the ensemble $\left\{\left|s_{i}\right\rangle, p_{i}\right\}_{i=0}^{2}$ is no easier than distinguishing between the two weighted states $\rho=p_{0}\left|s_{0}\right\rangle\left\langle s_{0}\right|$ and $\sigma=p_{1}\left|s_{1}\right\rangle\left\langle s_{1}\right|+$ $p_{2}\left|s_{2}\right\rangle\left\langle s_{2}\right|$. Indeed, any protocol distinguishing the three $\left|s_{i}\right\rangle$ can always be converted into a protocol for distinguishing $\rho$ and $\sigma$ by simply coarse-graining over all outcomes corresponding to $\left|s_{2}\right\rangle$ and $\left|s_{3}\right\rangle$. The minimum error probability in distinguishing $\rho$ and $\sigma$ is readily found to be (see Supplemental Material [14]):

$$
\frac{1}{2}-\frac{1}{2} \sqrt{1-3 p_{1} p_{2}-p_{0} p_{1}-p_{0} p_{2}},
$$

which, in the interval $-\pi / 6 \leqslant \theta<\pi / 6$, obtains a minimum of $1 / 2-\sqrt{3} / 4$. Now, this probability lower bounds the error 
probability along each branch of Alice's measurement, and therefore it places a lower bound on any one-way LOCC measurement scheme. In fact, this lower bound turns out to be tight. When Alice performs the POVM $\left\{\frac{2}{3}\left(\mathbb{I}-\left|s_{i}\right\rangle\left\langle s_{i}\right|\right)\right\}_{i=0}^{2}$ outcome $i$ will eliminate $\left|s_{i}\right\rangle \otimes\left|s_{i}\right\rangle$ but leave the other two states with an equal post-measurement probability. Thus, in each branch we obtain the error probability $1 / 2-\sqrt{3} / 4 \approx$ $6.70 \times 10^{-2}$, and this provides the minimum one-way error probability.

If we allow feedback from Bob, there exists better measurement strategies. The following protocol generalizes the optimal one-way scheme just described. Round I: Alice performs the measurement with Kraus operators given by $\left\{A_{i}\right\}_{i=0}^{2}$ with

$$
A_{i}=\sqrt{1 / 3(1-p)}\left|s_{i}\right\rangle\left\langle s_{i}|+\sqrt{1 / 3(1+p)}| s_{i}^{\perp}\right\rangle\left\langle s_{i}^{\perp}\right| .
$$

Here $\left|s_{i}^{\perp}\right\rangle$ is the state orthogonal to $\left|s_{i}\right\rangle$ (explicitly $\left|s_{i}^{\perp}\right\rangle=$ $\left.U^{i}|1\rangle\right)$. Note that this is the square root of the POVM given by Peres and Wootters [1]. Without loss of generality, we suppose that Alice obtains outcome " 0 " and communicates the result to Bob. Her (normalized) post-measurement states are $\left|s_{0}^{\prime}\right\rangle=$ $|0\rangle,\left|s_{1}^{\prime}\right\rangle=[2(2+p)]^{-1 / 2}(\sqrt{1-p}|0\rangle-\sqrt{3(1+p)}|1\rangle)$, and $\left|s_{2}^{\prime}\right\rangle=[2(2+p)]^{-1 / 2}(\sqrt{1-p}|0\rangle+\sqrt{3(1+p)}|1\rangle)$. Round II: From Bob's perspective, he is still dealing with the original states $\left|s_{i}\right\rangle$, but now their prior probabilities have changed to $P_{i \mid A_{0}}=P_{A_{0} \mid i}$. He now proceeds as if Alice had completely eliminated the state $\left|s_{0}\right\rangle$ (i.e., if she had chosen $p=1$ as the strength of her measurement). Specifically, he projects onto the eigenbasis of $\left|s_{1}\right\rangle\left\langle s_{1}|-| s_{2}\right\rangle\left\langle s_{2}\right|$ which are the states $| \pm\rangle=\sqrt{1 / 2}(|0\rangle \pm|1\rangle)$. A “+" outcome is associated with $\left|s_{1}\right\rangle$ and a "-" outcome is associated with $\left|s_{2}\right\rangle$; this is the optimal measurement for distinguishing between two pure states [17]. By the symmetry of the states, it is sufficient to only consider the "+" outcome, which he communicates to Alice. The conditional probabilities are $P_{A_{0} B_{+} \mid 0}=(1-p) / 6, P_{A_{0} B_{+} \mid 1}=$ $1 / 24(2+\sqrt{3})(2+p)$, and $P_{A_{0} B_{+} \mid 2}=1 / 24(2-\sqrt{3})(2+p)$. These can be inverted to give $P_{i \mid A_{0} B_{+}}=2 P_{A_{0} B_{+} \mid i}$. Round III: At this point, Alice still has three distinct states $\left|s_{0}^{\prime}\right\rangle,\left|s_{1}^{\prime}\right\rangle$, and $\left|s_{2}^{\prime}\right\rangle$. Here, $\left|s_{1}^{\prime}\right\rangle$ will have the greatest probability, while $\left|s_{0}^{\prime}\right\rangle$ will have the smallest when $p$ is close to 1 . Alice then ignores $\left|s_{2}^{\prime}\right\rangle$ and performs optimal discrimination between just $\left|s_{0}^{\prime}\right\rangle$ and $\left|s_{1}^{\prime}\right\rangle$. Letting $Q=P_{0 \mid A_{0} B_{+}}+P_{1 \mid A_{0} B_{+}}$, the minimum error probability is given by the well-known Helstrom bound [17] with normalized probabilities:

$$
P_{\text {err }}^{\left(A_{0} B_{+}\right)}=1-\frac{Q}{2}\left(1+\sqrt{1-4 \frac{P_{0 \mid A_{0} B_{+}} P_{1 \mid A_{0} B_{+}}}{Q^{2}}\left|\left\langle s_{0}^{\prime} \mid s_{1}^{\prime}\right\rangle\right|^{2}}\right) .
$$

By symmetry, each sequence of outcomes $\left(A_{i}, B_{\mu}\right)$ —with $i \in$ $\{0,1,2\}, \mu \in\{+,-\}$ - occurs with the same probability. Hence, the total error probability across all branches is given by $P_{\mathrm{err}}=$ $6 P_{\mathrm{err}}^{\left(A_{0} B_{+}\right)}$. The plot is given in Fig. 1. It obtains a minimum of approximately $6.47 \times 10^{-2}$, which is smaller than the one-way optimal of $1 / 2-\sqrt{3} / 4 \approx 6.70 \times 10^{-2}$. The one-way optimal probability is obtained at the point $p=1$.

Discussion and conclusions. Our results for minimum error discrimination of the double trine ensemble can be summarized as

$$
\mathrm{GLOBAL}=\mathrm{SEP}>\mathrm{LOCC}>\mathrm{LOCC}_{\rightarrow} .
$$

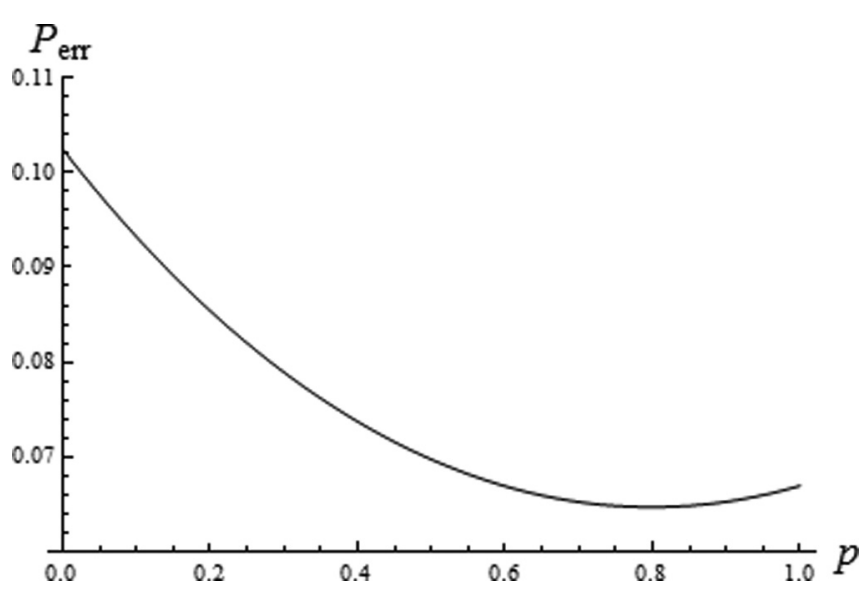

FIG. 1. The error probability $P_{\text {err }}$ using the above protocol as a function of Alice's measurement strength $p$. The point $p=1$ is the one-way minimum error probability.

We thus put substantial closure to a problem first posed over 20 years ago. A primary motivation for studying this problem is to better understand the limitations of processing quantum information by LOCC. Our results complement a series of recent results in this direction $[9,16,18]$. Theorem 1 largely extends the work of Ref. [16] as we reduce asymptotic minimum error discrimination of linearly independent states to asymptotic perfect discrimination. Asymptotic LOCC is not just a technical consideration, but rather it arises from very practical situations. Since any experiment has an unavoidable margin of error, there is effectively no difference between perfect distinguishability and asymptotically perfect distinguishability. Hence, any no-go LOCC distinguishability result only becomes relevant if it is a "robust" phenomenon under small uncertainties. In other words, the no-go result must also hold for asymptotic LOCC, and this is exactly what we prove for the double trine ensemble.

We note that $\mathrm{C} 1$ and $\mathrm{C} 2$ have been previously observed using contrived distinguishability measures, higher-dimensional systems, and/or mixed-state ensembles [19-21]. Our work is distinct from all previous results in that it deals with pure twoqubit states and minimum error probability, a highly natural measure of distinguishability. The fact that we consider pure ensembles with three states is significant since it is well-known that any two pure states can be distinguished optimally via LOCC (i.e., LOCC = GLOBAL) [22,23]. Thus, with the double trine being a real, symmetric, and pure ensemble of two qubits, we have identified the simplest type of ensemble in which $\mathrm{LOCC} \neq \mathrm{GLOBAL}$ for state discrimination.

Furthermore, since the double trine ensemble consists of product states (i.e., no entanglement), it demonstrates the phenomenon of "nonlocality without entanglement" (NLWE). This distinction is further sharpened by considering that LOCC $\neq$ SEP for the optimal discrimination of the double trine. Separable operations are interesting since, like LOCC operations, they lack the ability to create entanglement. Nevertheless, SEP evidently possesses some nonlocal power as it can outperform LOCC in discriminating the double trine. This Rapid Communication offers an example of NLWE in two-qubit pure state ensembles, whereas all previous examples 
focus on $3 \otimes 3$ systems or larger. One should not take for granted the fact that NLWE exists in both $2 \otimes 2$ and $3 \otimes 3$ systems since we know entanglement behaves quite differently in these dimensions (consider, for instance, bound entanglement). Thus, NLWE is a very fundamental physical phenomenon that emerges in even the most basic multipart quantum systems.
Acknowledgments. We would like to thank Runyao Duan, Debbie Leung, and Laura Mančinska for helpful discussions on the topic of LOCC distinguishability. We especially thank Sarah Croke for pointing out an error in a previous version of the paper. M.H. acknowledges financial support from the Vice Chancellor's Postdoctoral Research Fellowship, University of Technology, Sydney.
[1] A. Peres and W. K. Wootters, Phys. Rev. Lett. 66, 1119 (1991).

[2] A. Holevo, J. Multivariate Anal. 3, 337 (1973); M. A. Nielsen and I. L. Chuang, Quantum Computation and Quantum Information (Cambridge University Press, Cambridge, UK, 2000).

[3] C. H. Bennett, G. Brassard, C. Crépeau, R. Jozsa, A. Peres, and W. K. Wootters, Phys. Rev. Lett. 70, 1895 (1993).

[4] C. H. Bennett, D. P. DiVincenzo, C. A. Fuchs, T. Mor, E. Rains, P. W. Shor, J. A. Smolin, and W. K. Wootters, Phys. Rev. A 59, 1070 (1999).

[5] B. M. Terhal, D. P. DiVincenzo, and D. W. Leung, Phys. Rev. Lett. 86, 5807 (2001); D. P. DiVincenzo, D. W. Leung, and B. M. Terhal, IEEE Trans. Inf. Theory 48, 580 (2002).

[6] R. Horodecki, P. Horodecki, M. Horodecki, and K. Horodecki, Rev. Mod. Phys. 81, 865 (2009).

[7] W. K. Wootters, Int. J. Quantum Inf. 4, 219 (2006).

[8] E. Chitambar, W. Cui, and H.-K. Lo, Phys. Rev. Lett. 108, 240504 (2012).

[9] E. Chitambar, D. Leung, L. Mančinska, M. Ozols, and A. Winter, arXiv: 1210.4583.

[10] Recall that the "pretty good measurement" for an ensemble $\left\{\left|\phi_{i}\right\rangle, p_{i}\right\}_{i=1}^{k}$ is the POVM with elements $p_{i} \rho^{-1 / 2}\left|\phi_{i}\right\rangle\left\langle\phi_{i}\right| \rho^{-1 / 2}$, where $\rho=\sum_{i=1}^{k} p_{i}\left|\phi_{i}\right\rangle\left\langle\phi_{i}\right|$ [24].
[11] M. Ban, K. Kurokawa, R. Momose, and O. Hirota, Int. J. Theor. Phys. 36, 1269 (1997).

[12] H. Yuen, R. Kennedy, and M. Lax, IEEE Trans. Inf. Theory 21, 125 (1975).

[13] C. Mochon, Phys. Rev. A 73, 032328 (2006).

[14] See Supplemental Material at http://link.aps.org/supplemental/ 10.1103/PhysRevA.88.020302.

[15] J. Walgate and L. Hardy, Phys. Rev. Lett. 89, 147901 (2002).

[16] M. Kleinmann, H. Kampermann, and D. Bruß, Phys. Rev. A 84, 042326 (2011)

[17] C. W. Helstrom, Quantum Detection and Estimation Theory (Academic Press, New York, 1976).

[18] A. M. Childs, D. Leung, L. Mančinska, and M. Ozols, arXiv:1206.5822.

[19] S. Massar and S. Popescu, Phys. Rev. Lett. 74, 1259 (1995).

[20] M. Koashi, F. Takenaga, T. Yamamoto, and N. Imoto, arXiv:0709.3196.

[21] M. Owari and M. Hayashi, New J. Phys. 10, 013006 (2008).

[22] J. Walgate, A. J. Short, L. Hardy, and V. Vedral, Phys. Rev. Lett. 85, 4972 (2000).

[23] S. Virmani, M. F. Sacchi, M. B. Plenio, and D. Markham, Phys. Lett. A 288, 62 (2001).

[24] P. Hausladen and W. K. Wootters, J. Mod. Opt. 41, 2385 (1994). 